\title{
Abnormal Glucose Tolerance in Prediabetes Patients with Acute Myocardial Infarction: Implications for Therapy

\author{
Nitchakarn Laichuthai ${ }^{1}$, Ralph A. DeFronzo ${ }^{2 *}$
}

'Hormonal and Metabolic Disorders Research Unit and Excellence Center in Diabetes, Hormone, and Metabolism, King Chulalongkorn Memorial Hospital, Thai Red Cross Society, and Division of Endocrinology and Metabolism, Department of Medicine, Faculty of Medicine, Chulalongkorn University, Bangkok, Thailand 2Diabetes Division, UT Health San Antonio and Texas Diabetes Institute, San Antonio, Texas, USA

Article Info

\section{Article Notes}

Received: January 25,2021

Accepted: March 26, 2021

\section{*Correspondence:}

${ }^{*}$ Dr. Ralph A. DeFronzo, Diabetes Division, UT Health San Antonio and Texas Diabetes Institute, San Antonio, Texas, USA; Telephone No: 210-567-6691:

Email: defronzo@uthscsa.edu.

${ }^{\circledR} 2021$ DeFronzo RA. This article is distributed under the terms of the Creative Commons Attribution 4.0 International License.

\section{Keywords:}

Abnormal glucose tolerance

Acute myocardial infarction

Recurrent cardiovascular events

Prediabetes

Type 2 diabetes

\section{Abstract}

Newly discovered abnormal glucose tolerance is common in patients who present with acute myocardial infarction (MI). These individuals are at very high risk for recurrent major adverse cardiovascular events (MACE), cardiovascular (CV) mortality, and all-cause mortality compared to normal-glucose-tolerant individuals who present with acute MI. Early and aggressive intervention with lifestyle and pharmacologic treatment are essential for the prevention of prediabetes progression to diabetes and recurrent cardiovascular events in this high risk population. Management, both with regard to prevention of recurrent cardiovascular events and development of diabetes, has been poorly addressed in current cardiology and diabetes guidelines. In this article, we review current evidence regarding the use of glucagon-like peptide 1 receptor agonists (GLP-1 RAs), sodium glucose cotransporter 2 inhibitors (SGLT2i), and pioglitazone to prevent recurrent cardiovascular events and propose areas of research to be explored in the future.

\section{Introduction}

Type 2 diabetes mellitus (T2DM) is a cardiorenal-metabolic disease characterized by defects in both insulin secretion and insulin sensitivity ${ }^{1}$. Diabetes mellitus is a strong risk factor for atherosclerotic cardiovascular disease (ASCVD) and is associated with a 2- to 4- fold higher cardiovascular (CV) mortality rate compared to nondiabetic individuals in the general population in every age group $^{2-4}$. Even with the treatment of established cardiovascular (CV) risk factors, including hypertension, dyslipidemia, prothrombotic state, diabetes mellitus remains significantly associated with increased all-cause and CV mortality ${ }^{5}$.

Abnormal glucose tolerance encompasses a group of individuals with impaired fasting glucose (IFG), impaired glucose tolerance (IGT), and overt diabetes. Individuals with IFG and IGT are referred to as having prediabetes ${ }^{6}$. Prediabetes also can be defined by the HbA1c $=5.7-6.4 \%{ }^{6}$. People with prediabetes are maximally $/$ near maximally insulin resistant ${ }^{1}$ and have an increased risk for cardiovascular disease $\mathrm{e}^{7-9}$. They also manifest impaired beta cell function, although the defect in insulin secretion is not as severe as in individuals with type 2 diabetes ${ }^{1}$. In patients with T2DM there is a strong relationship between the 2-hour plasma glucose concentration and $\mathrm{CV}$ risk that begins at a range below the threshold for diabetes diagnosis ${ }^{9}$. Insulin resistance also is strongly related with cardiovascular disease, independently of the state of glucose tolerance $\mathrm{e}^{10-13}$. The relationship between insulin resistance and 
atherosclerotic cardiovascular disease persists even after adjustment for confounding factors, including glucose tolerance status, plasma lipid profile, smoking, hypertension, and $\mathrm{BMI}^{10-13}$. Insulin resistance also is an independent predictor of increased risk of CVD in nondiabetic subjects ${ }^{13,14}$. Three mechanisms account for the association between insulin resistance and ASCVD: (i) the basic molecular etiology of the insulin resistance which leads to impaired nitric oxide generation and increased stimulation of the MAP kinase or atherogenic pathway ${ }^{10}$, (ii) the compensatory hyperinsulinemia ${ }^{14}$, and (iii) the association between insulin resistance and features of the metabolic syndrome ${ }^{10}$ (Figure 1).

\section{Newly Discovered Abnormal Glucose Tolerance in Patients with Acute MI and Risk of Recurrent ASCVD and Mortality}

Previously undiagnosed diabetes and impaired glucose tolerance are common in patients with acute myocardial infarction. In a recent meta-analysis involving 19 studies with 41, 509 individuals and follow-up of 3.1 years $^{15}$, we demonstrated that the prevalence of newly discovered abnormal glucose tolerance (prediabetes and diabetes) in patients with acute MI was 48\%. Moreover, individuals with newly diagnosed abnormal glucose tolerance and acute MI had a 1.5-fold increased risk of all-cause mortality and 1.4-fold increased risk of recurrent MACE (Major Adverse Cardiovascular Events). The increased CV risk in prediabetes subjects was as high as that observed in patients with type 2 diabetes mellitus ${ }^{15}$. These results clearly demonstrate that the increased CV risk in prediabetic individuals cannot be explained by hyperglycemia and most likely is attributed to the underlying insulin resistance and associated cardiovascular risk factors. Early detection of abnormal glucose tolerance, i.e., prediabetes or diabetes, in patients with acute myocardial infarction and aggressive secondary risk reduction strategies is essential in order to reduce the rate of recurrent CV complications ${ }^{16,17}$ and development of type 2 diabetes mellitus (T2DM) ${ }^{18}$.

Glucose tolerance status most commonly is evaluated by one of three tests: fasting plasma glucose concentration, HbA1c, or 75-gram OGTT. In patients with acute MI, the OGTT has been used to classify the glucose tolerance status in the majority of published studies ${ }^{15}$. The OGTT has higher sensitivity to detect abnormal glucose tolerance, but it is more time-consuming. Moreover, the OGTT can be confounded by stress associated with acute MI and is not reliable until at least 5-7 days after myocardial infarction. HbA1c measurement is particularly useful since it is not

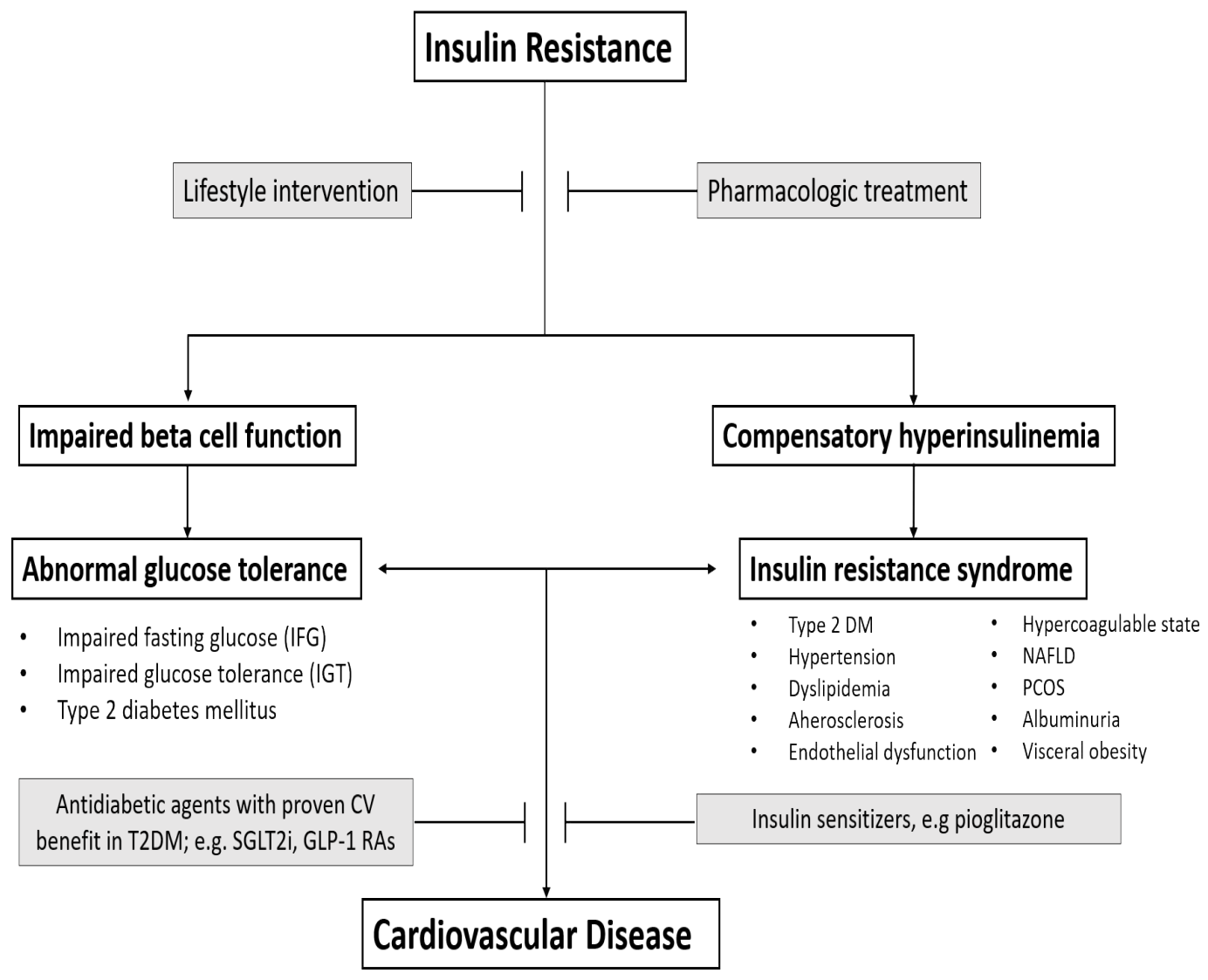

Figure 1: Relationship between insulin resistance, abnormal glucose tolerance, and cardiovascular disease. A pathophysiologicbased approach is presented in grey boxes. 
affected by the stress of an acute MI. However, the HbA1c is less sensitive than the OGTT and many patients with a $\mathrm{HbA1c}$ in the $6.0-6.4 \%$ range will turn out to have overt diabetes during the OGTT (2-hour PG $\geq 200 \mathrm{mg} / \mathrm{dl}$ ). Evaluation of glycemic status by measuring the fasting plasma glucose concentration and HbA1c when the patient is hospitalized with acute MI followed by an OGTT 1-2 weeks later to define whether the patient has overt diabetes or prediabetes (impaired glucose tolerance and/ or impaired fasting glucose) or has reverted to normal glucose tolerance represents a pragmatic approach.

\section{Management of Newly Discovered Abnormal Glucose Tolerance in Acute MI Patients}

Because of the high risk of recurrent cardiovascular events in acute MI patients with newly discovered abnormal glucose tolerance ${ }^{15}$, early and aggressive intervention, both lifestyle and pharmacologic, is important for the prevention of both diabetes and recurrent cardiovascular events in this high CV risk population. In patients with diabetes, management strategies are well-established. However, for people with prediabetes, management guidelines have not been clearly defined and this group of patients often receives less aggressive management of CV risk factors. As discussed earlier, insulin resistance is an important pathophysiologic abnormality which contributes to the accelerated cardiovascular disease in diabetes and prediabetes patients. It can be assumed, therefore, that individuals who present with an acute MI and abnormal glucose tolerance are maximally/near-maximally insulin resistant. In these prediabetes individuals, the conventional treatment approach does not address the underlying insulin resistance, and there are no specific guidelines concerning the use of specific glucose-lowering agents, e.g., SGLT2i and GLP1-RA, which clearly have been shown to have $\mathrm{CV}$ benefit in T2DM individuals ${ }^{19,20}$. For people with prediabetes, early and aggressive lifestyle intervention to prevent the progression to diabetes is as important as the standard medical treatment for coronary artery disease (antiplatelet drugs, RAAS blockade, statins, beta-blockers). The principles of management is outlined in Figure 1.

\section{Lifestyle Intervention and Risk Factors Management}

Lifestyle intervention and risk factors modification represent the cornerstones for the management of all cardio-metabolic diseases. In the Steno-2 study, intensive therapy with multiple drug combinations and behavior modification was shown to be superior to conventional therapy in terms of reduced CV complications and rates of death from any cause and cardiovascular death in patients with $\mathrm{T}_{2} \mathrm{DM}^{16}$. In a recently published study, the excess risk of death and cardiovascular events in patients with T2DM could be reduced by optimizing 5 risk factors, including glycated hemoglobin, LDL cholesterol, blood pressure, albuminuria, and smoking status, to be within target ranges ${ }^{21}$.

In diabetic, as well as nondiabetic, patients with acute coronary syndrome (ACS) all guidelines recommend RAAS blockers, statins, and antiplatelet therapy to prevent recurrent CV events. In diabetic patients with an established CV event, SGLT2i and GLP-1 receptor agonists are recommended as first line therapy for prevention of recurrent $\mathrm{CV}$ events and glycemic control by the European Cardiology Society (ECS) ${ }^{22}$, while the ADA/EASD recommends that a GLP-1 RA or SGLT2i be the second drug following metformin ${ }^{23}$. The authors strongly favor the ECS approach. However, for people with prediabetes, e.g., impaired fasting glucose or impaired glucose tolerance, there are no specific guidelines for pharmacologic management. In this group it makes sense to initiate therapy with medications that have been shown to have proven $\mathrm{CV}$ benefit in diabetic patients with a previous $\mathrm{CV}$ event or with $\mathrm{CV}$ risk factors but without a prior $\mathrm{CV}$ event: GLP-1 RA, SGLT2i, pioglitazone ${ }^{12,19-24}$. These same medications also have been shown to slow the progression of prediabetes to diabetes ${ }^{25-27}$.

\section{GLP-1 Receptor Agonists}

GLP-1 RAs reduce HbA1c, promote weight loss, have a low risk of hypoglycemia, and correct 6 of the 8 components of the Ominous Octet ${ }^{1}$. In addition, liraglutide (LEADER) $(\mathrm{HR}=0.87,95 \% \mathrm{CI}=0.78-0.97, \mathrm{p}=0.01)^{28}$, semaglutide $\left(\right.$ SUSTAIN-6) $(\mathrm{HR}=0.74,95 \% \mathrm{CI}=0.58-0.95, \mathrm{p}=0.02){ }^{29}$, albiglutide (HARMONY) (HR $=0.78 ; 95 \% \mathrm{CI}=0.68-0.90$, $\mathrm{p}=0.0006){ }^{30}$, exenatide (EXSCEL) (HR=0.91, 95\% CI $=0.83-$ $1.00, \mathrm{p}=0.06)^{31}$, and dulaglutide (REWIND) (HR=0.88, 95\% $\mathrm{CI}=0.79-0.99, \mathrm{p}=0.026){ }^{32}$ have been shown to decrease 3-point MACE. Of note, the CV benefit of dulaglutide in REWIND $^{32}$ was observed in individuals with a previous $\mathrm{CV}$ event as well as in individuals with $\mathrm{CV}$ risk factors but without a prior CV event.

\section{SGTL2 Inhibitors}

SGLT2i promote glucosuria in the amount of 70 80 grams/day by inhibiting glucose reabsorption in the proximal tubule. They effectively lower the HbA1c, promote weight loss, reduce blood pressure, decrease arterial stiffness, and do not cause hypoglycemia ${ }^{33}$. Because they work on the kidney, they can be used in combination with all other antidiabetic agents. SGLT2 inhibitors also have been demonstrated to decrease three-point MACE, hospitalization for heart failure, and, for some agents in the class, CV death in T2DM individuals with established ASCVD $^{34-36}$. Although some differences in CV outcomes have been observed between various SGLT2 inhibitors in published CVOTs, these differences are more related to differences in the patient population than to intrinsic 
differences between the individual SGLT2i drugs ${ }^{19}$. Thus, in EMPA-REG OUTCOME (empagliflozin), over 99\% of participants had a previous CV event and it, therefore, is not surprising that decreased CV mortality was the primary driver of 3-point MACE (HR $=0.86,95 \%$ CI $=0.74-0.99$, $\mathrm{p}=0.04)^{34}$. Empagliflozin also reduced hospitalization for heart failure and the renal composite outcome ${ }^{34}$. In the CANVAS PROGRAM ${ }^{35}$ canagliflozin decreased 3-point MACE ( $\mathrm{HR}=0.86,95 \% \mathrm{CI}=0.75-0.97, \mathrm{p}=0.02)$, as well as heart failure hospitalization and the renal composite outcome. In DECLARE-TIMI, patients randomized to dapagliflozin had a $17 \%$ reduction $(\mathrm{p}<0.005)$ in the primary composite outcome of $\mathrm{CV}$ death plus hospitalization for heart failure but no difference in 3-point MACE ${ }^{36}$. However, the majority of patients $(\sim 60 \%)$ in DECLARE-TIMI had CV risk factors but no prior CV event; thus, DECLARE-TIMI had the least sick population. Dapagliflozin also reduced the composite renal outcome by $24 \%{ }^{36}$. In VERTIS-CV ertugliflozin did not cause a significant reduction in 3-point MACE, but hospitalization for heart failure decreased (not tested for statistical significance) ${ }^{37}$. In DAPA-HF ${ }^{38}$ and EMPEROR ${ }^{39}$, dapagliflozin and empagliflozin, respectively, significantly reduced $\mathrm{HHF}$ and $\mathrm{CV}$ mortality in both diabetic and nondiabetic individuals with heart failure and reduced ejection fraction. Two SGLT2 inhibitors, canagliflozin (CREDENCE) $^{40}$ and dapagliflozin (DAPA-CKD) ${ }^{41}$, have been shown to reduce the composite renal outcome in diabetic ${ }^{40}$ as well in nondiabetic and diabetic subjects ${ }^{41}$.

\section{Thiazolidinediones}

The insulin-sensitizing agent, pioglitazone, has been overlooked in the era of GLP-1 RAs and SGLT2 inhibitors. In the IRIS trial, pioglitazone reduced recurrent stroke and CV events and progression to diabetes in insulin resistant, nondiabetic subjects with a recent (within 6 months) stroke or transient ischemic attack ${ }^{12}$. In the PROactive trial ${ }^{24}, 5238$ T2DM patients with a prior CV event were randomized to pioglitazone or placebo. Although the primary endpoint, which included MACE and leg revascularization, did not reach statistical significance $(H R=0.90, p=0.09)$, the main secondary endpoint, MACE, was significantly reduced ( $\mathrm{HR}=0.84, \mathrm{p}=0.027)$ with an effect size similar to that in recent cardiovascular outcome trials with GLP-1 RAs and SGLT2 inhibitors. Pioglitazone reduced recurrent MI by $25 \%$ and recurrent stroke by $47 \%{ }^{24}$. Pioglitazone also has been shown to slow the progression of carotid intimal media thickness in the Chicago Study ${ }^{42}$ and to reduce coronary artery plaque volume in the PERISCOPE study $^{43}$, independent of improvement in hyperglycemia, dyslipidemia, or blood pressure, suggesting a direct vascular benefit of the thiazolidinedione.

Pioglitazone also has a number of other benefits, including amelioration of insulin resistance, preservation of beta-cell function, durable glycemic control, improvement of multiple factors of the metabolic syndrome and reversal of hepatic steatosis and fibrosis ${ }^{1,10,44}$. Further, pioglitazone is generically available, making it an attractive option for the treatment of many patients with T2DM.

\section{Metformin}

Metformin is safe and has been in long standing use worldwide. In the Diabetes Prevention Program (DPP), metformin decreased the incidence of type 2 diabetes by $35 \%$ compared to placebo in prediabetes individuals ${ }^{18}$. For treatment of T2DM, metformin is recommended as first line therapy by the ADA/EASD ${ }^{23}$. Contrary to common belief, metformin is not an insulin sensitizer in muscle ${ }^{45}$. Metformin enters cells via the organic cation transporter (OTC) and the OTC is not present in skeletal or cardiac muscle $^{46}$. The major mechanism of action of metformin to improve glycemic control in T2DM is mediated via inhibition of gluconeogenesis and subsequent reduction in hepatic glucose production ${ }^{45,47}$. Metformin also has no effect on the beta cell to improve insulin secretion ${ }^{45}$, explaining the progressive rise in $\mathrm{HbA1c}$ after the first year of initiation of therapy ${ }^{48,49}$. Further, no large long-term prospective study has demonstrated that metformin reduces $\mathrm{CV}$ events in diabetic patients and the European Cardiology Society has recommended that GLP-1 RAs or SGLT2i be used as first line therapy (not metformin) in diabetic patients with an established CV event ${ }^{50}$. The present authors strongly favor the ECS approach.

Role of GLP1-RAs, SGLT2i and Pioglitazone in Patients with Abnormal Glucose Tolerance after Acute Myocardial Infarction

No previous CVOT has evaluated the effect of any antidiabetic agent in prediabetes patients with an acute MI. In the IRIS trial ${ }^{12}$ insulin resistant individuals (HOMAIR $>3.0$ and mean $\mathrm{HbA} 1 \mathrm{c}=5.8 \pm 0.4 \%$ ) with a recent history of stroke or TIA were randomized to treatment with pioglitazone or placebo. Pioglitazone-treated subjects experienced a $24 \%$ reduction in recurrent CV events and a $52 \%$ decrease in development of diabetes over a 4.8 year follow up. Obviously, in IRIS ${ }^{12}$ and in other studies in prediabetic individuals ${ }^{51}$ the cardiovascular benefit cannot be explained by a reduction in the plasma glucose concentration. In DAPA- $\mathrm{HF}^{38}$, dapagliflozin significantly reduced cardiovascular events and also decreased the development of diabetes in non-diabetic individuals by $32 \%(\mathrm{P}<0.019)$. Liraglutide ${ }^{27}$ also has been shown to markedly reduce the development of diabetes in prediabetic individuals but no study with any GLP-1 RA has examined the impact on $\mathrm{CV}$ prevention in this population. Thus, there is convincing data that: (i) pioglitazone and GLP-1 RA, and possibly SGLT2i, decrease the conversion of prediabetes to diabetes, and (ii) that pioglitazone decreases CV events in a prediabetic population. ASCVD is well established in 
prediabetic individuals, long before the diagnosis of type 2 diabetes. Therefore, it is reasonable to assume that the CV benefits of GLP-1 RAs and SGLT2 inhibitors also would be seen in prediabetic patients with a recent MI and the authors recommend aggressive treatment with a GLP-1 RA, SGLT2i, pioglitazone, or some combination thereof to reduce the risk of recurrent events in this high risk group. A large prospective CVOT is warranted to examine the most effective pharmacologic intervention to prevent recurrent $\mathrm{CV}$ events in this large population of individuals who present with an acute MI and subsequently are found to have prediabetes.

\section{Conclusion}

Abnormal glucose tolerance, including prediabetes and diabetes, is common in patients who present with acute myocardial infarction and are previously unknown to have any disturbance in glucose homeostasis. These individuals are at very high risk for recurrent MACE and CV mortality. Early and aggressive intervention, both lifestyle and pharmacologic, is important for the prevention of recurrent cardiovascular events and progression of prediabetes to diabetes.

\section{References}

1. DeFronzo RA. Banting Lecture. From the triumvirate to the ominous octet: a new paradigm for the treatment of type 2 diabetes mellitus. Diabetes. 2009; 58(4): 773-95.

2. Bertoni AG, Krop JS, Anderson GF, et al. Diabetes-related morbidity and mortality in a national sample of U.S. elders. Diabetes Care. 2002; 25(3): 471-5.

3. Kannel WB, McGee DL. Diabetes and cardiovascular disease. The Framingham study. JAMA. 1979; 241(19): 2035-8.

4. Haffner SM, Lehto $S$, Ronnemaa $\mathrm{T}$, et al. Mortality from coronary heart disease in subjects with type 2 diabetes and in nondiabetic subjects with and without prior myocardial infarction. N Engl J Med. 1998; 339(4): 229-34.

5. Raghavan S, Vassy JL, Ho YL, et al. Diabetes Mellitus-Related All-Cause and Cardiovascular Mortality in a National Cohort of Adults. J Am Heart Assoc. 2019; 8(4): e011295.

6. American Diabetes Association 2. Classification and Diagnosis of Diabetes: Standards of Medical Care in Diabetes-2021. Diabetes Care. 2021; 44(Suppl 1): S15-S33.

7. Huang Y, Cai X, Mai W, et al. Association between prediabetes and risk of cardiovascular disease and all cause mortality: systematic review and meta-analysis. BMJ. 2016; 355: i5953.

8. Haffner SM, Stern MP, Hazuda HP, et al. Cardiovascular risk factors in confirmed prediabetic individuals. Does the clock for coronary heart disease start ticking before the onset of clinical diabetes? JAMA. 1990; 263(21): 2893-8.

9. Chattopadhyay S, George A, John J, et al. Adjustment of the GRACE score by 2-hour post-load glucose improves prediction of long-term major adverse cardiac events in acute coronary syndrome in patients without known diabetes. Eur Heart J. 2018; 39(29): 2740-5.

10. DeFronzo RA. Insulin resistance, lipotoxicity, type 2 diabetes and atherosclerosis: the missing links. The Claude Bernard Lecture 2009. Diabetologia 2010; 53(7): 1270-1287.
11. Hanley AJ, Williams K, Stern MP, et al. Homeostasis model assessment of insulin resistance in relation to the incidence of cardiovascular disease: the San Antonio Heart Study. Diabetes Care. 2002; 25(7): 1177-84.

12. Kernan WN, Viscoli CM, Furie KL, et al. Pioglitazone after Ischemic Stroke or Transient Ischemic Attack. N Engl J Med. 2016; 374(14): 1321-31

13. Gast KB, Tjeerdema N, Stijnen T, et al. Insulin resistance and risk of incident cardiovascular events in adults without diabetes: metaanalysis. PLoS One. 2012; 7(12): e52036.

14. Ferrannini E, Balkau B, Coppack SW, et al. Insulin resistance, insulin response, and obesity as indicators of metabolic risk. J Clin Endocrinol Metab 2007; 92(8): 2885-2892.

15. Laichuthai N, Abdul-Ghani M, Kosiborod M, et al. Newly Discovered Abnormal Glucose Tolerance in Patients With Acute Myocardial Infarction and Cardiovascular Outcomes: A Meta-analysis. Diabetes Care. 2020; 43(8): 1958-66.

16. Gaede P, Lund-Andersen H, Parving HH, et al. Effect of a multifactorial intervention on mortality in type 2 diabetes. N Engl J Med. 2008; 358(6): 580-91.

17. Ferrannini E, DeFronzo RA. Impact of glucose-lowering drugs on cardiovascular disease in type 2 diabetes. Eur Heart J. 2015; 36(34): 2288-2296

18. Knowler WC, Barrett-Connor E, Fowler SE, et al. Reduction in the incidence of type 2 diabetes with lifestyle intervention or metformin. N Engl J Med. 2002; 346(6): 393-403.

19. McGuire DK, Shih WJ, Cosentino F, et al. Association of SGLT2 Inhibitors With Cardiovascular and Kidney Outcomes in Patients With Type 2 Diabetes: A Meta-analysis. JAMA Cardiol. 2021; 6(2): 148-158.

20. Kristensen SL, Rorth R, Jhund PS, et al. Cardiovascular, mortality, and kidney outcomes with GLP-1 receptor agonists in patients with type 2 diabetes: a systematic review and meta-analysis of cardiovascular outcome trials. Lancet Diabetes Endocrinol. 2019; 7(10): 776-785.

21. Rawshani A, Rawshani A, Franzen S, et al. Risk Factors, Mortality, and Cardiovascular Outcomes in Patients with Type 2 Diabetes. N Engl J Med. 2018; 379(7): 633-44.

22. Collet JP, Thiele H, Barbato E, et al. 2020 ESC Guidelines for the management of acute coronary syndromes in patients presenting without persistent ST-segment elevation. Eur Heart J. 2020.

23. American Diabetes Association. 9. Pharmacologic Approaches to Glycemic Treatment: Standards of Medical Care in Diabetes-2021. Diabetes Care. 2021; 44(Suppl 1): S111-S24.

24. Dormandy JA, Charbonnel B, Eckland DJ, et al. Secondary prevention of macrovascular events in patients with type 2 diabetes in the PROactive Study (PROspective pioglitAzone Clinical Trial In macroVascular Events): a randomised controlled trial. Lancet. 2005; 366(9493): 1279-89.

25. DeFronzo RA, Tripathy D, Schwenke DC, et al. Pioglitazone for diabetes prevention in impaired glucose tolerance. $\mathrm{N}$ Engl J Med. 2011; 364(12): 1104-1115.

26. Inzucchi SE, Fitchett D, Jurisic-Erzen D, et al. Are the cardiovascular and kidney benefits of empagliflozin influenced by baseline glucoselowering therapy? Diabetes Obes Metab. 2020; 22(4): 631-639.

27. le Roux CW, Astrup A, Fujioka K, et al. 3 years of liraglutide versus placebo for type 2 diabetes risk reduction and weight management in individuals with prediabetes: a randomised, double-blind trial. Lancet. 2017; 389(10077): 1399-409.

28. Marso SP, Daniels GH, Brown-Frandsen $\mathrm{K}$, et al. Liraglutide and Cardiovascular Outcomes in Type 2 Diabetes. N Engl J Med. 2016; 375(4): 311-22. 
29. Marso SP, Bain SC, Consoli A, et al. Semaglutide and Cardiovascular Outcomes in Patients with Type 2 Diabetes. N Engl J Med. 2016; 375(19): 1834-44.

30. Hernandez AF, Green JB, Janmohamed S, et al. Albiglutide and cardiovascular outcomes in patients with type 2 diabetes and cardiovascular disease (Harmony Outcomes): a double-blind, randomised placebo-controlled trial. Lancet. 2018; 392(10157): 1519-29.

31. Holman RR, Bethel MA, Mentz RJ, et al. Effects of Once-Weekly Exenatide on Cardiovascular Outcomes in Type 2 Diabetes. N Engl J Med. 2017; 377(13): 1228-1239.

32. Gerstein HC, Colhoun HM, Dagenais GR, et al. Dulaglutide and cardiovascular outcomes in type 2 diabetes (REWIND): a double-blind, randomised placebo-controlled trial. Lancet. 2019; 394(10193): 12130.

33. DeFronzo RA, Norton L, Abdul-Ghani M. Renal, metabolic and cardiovascular considerations of SGLT2 inhibition. Nat Rev Nephrol. 2017; 13(1): 11-26.

34. Zinman B, Wanner C, Lachin JM, et al. Empagliflozin, Cardiovascular Outcomes, and Mortality in Type 2 Diabetes. N Engl J Med. 2015; 373(22): 2117-28.

35. Neal B, Perkovic V, Mahaffey KW. Canagliflozin and Cardiovascular and Renal Events in Type 2 Diabetes. N Engl J Med. 2017; 377(7): 644-657.

36. Wiviott SD, Raz I, Bonaca MP, et al. Dapagliflozin and Cardiovascular Outcomes in Type 2 Diabetes. N Engl J Med. 2019; 380(4): 347-357.

37. Cannon CP, Pratley R, Dagogo-Jack S, et al. Cardiovascular Outcomes with Ertugliflozin in Type 2 Diabetes. N Engl J Med. 2020; 383(15): 1425-35.

38. McMurray JJV, Docherty KF, Jhund PS. Dapagliflozin in Patients with Heart Failure and Reduced Ejection Fraction. Reply. N Engl J Med. 2020; 382(10): 973.

39. Packer M, Anker SD, Butler J, et al. Cardiovascular and Renal Outcomes with Empagliflozin in Heart Failure. N Engl J Med. 2020; 383(15): 1413-1424.
40. Perkovic V, Jardine MJ, Neal B, et al. Canagliflozin and Renal Outcomes in Type 2 Diabetes and Nephropathy. N Engl J Med. 2019; 380(24): 2295-306.

41. Heerspink HJL, Stefansson BV, Correa-Rotter R, et al. Dapagliflozin in Patients with Chronic Kidney Disease. N Engl J Med. 2020; 383(15): 1436-46.

42. Mazzone T, Meyer PM, Feinstein SB, et al. Effect of pioglitazone compared with glimepiride on carotid intima-media thickness in type 2 diabetes: a randomized trial. JAMA. 2006; 296(21): 2572-2581.

43. Nissen SE, Nicholls SJ, Wolski K, et al. Comparison of pioglitazone vs glimepiride on progression of coronary atherosclerosis in patients with type 2 diabetes: the PERISCOPE randomized controlled trial. JAMA. 2008; 299(13): 1561-1573.

44. Eldor R, DeFronzo RA, Abdul-Ghani M. In vivo actions of peroxisome proliferator-activated receptors: glycemic control, insulin sensitivity, and insulin secretion. Diabetes Care. 2013; 36(Suppl 2): S162-174.

45. Abdul-Ghani M, DeFronzo RA. Is It Time to Change the Type 2 Diabetes Treatment Paradigm? Yes! GLP-1 RAs Should Replace Metformin in the Type 2 Diabetes Algorithm. Diabetes Care. 2017; 40(8): 11211127.

46. Shu Y, Sheardown SA, Brown C, et al. Effect of genetic variation in the organic cation transporter 1 (OCT1) on metformin action. J Clin Invest. 2007; 117(5): 1422-31.

47. Cusi K, Consoli A, DeFronzo RA. Metabolic effects of metformin on glucose and lactate metabolism in noninsulin-dependent diabetes mellitus. J Clin Endocrinol Metab. 1996; 81(11): 4059-67.

48. Intensive blood-glucose control with sulphonylureas or insulin compared with conventional treatment and risk of complications in patients with type 2 diabetes (UKPDS 33). UK Prospective Diabetes Study (UKPDS) Group. Lancet. 1998; 352(9131): 837-853.

49. Brown JB, Conner C, Nichols GA. Secondary failure of metformin monotherapy in clinical practice. Diabetes Care. 2010; 33(3): 501-506.

50. Cosentino F, Grant PJ, Aboyans V, et al. 2019 ESC Guidelines on diabetes, pre-diabetes, and cardiovascular diseases developed in collaboration with the EASD. Eur Heart J. 2020; 41(2): 255-323. 